\title{
Estimativas da evapotranspiração pelos métodos de Thornthwaith e Penman-Monteith para a região de Taubaté, Vale do Paraíba.
}

\author{
Diogo Gadioli de Oliveira ${ }^{1}$ \\ Gilberto Fisch ${ }^{1}$ \\ Maria Cristina Forti ${ }^{2}$ \\ ${ }^{1}$ Universidade de Taubaté - UNITAU \\ Estrada Mun. Dr. José Luiz Cembranelli, 5.000- 12080-010 - Taubaté - SP, Brasil \\ digadioli@hotmail.com,gfisch@iae.cta.br \\ ${ }^{2}$ Instituto Nacional de Pesquisas Espaciais - INPE \\ Av. do Astronautas, 1758 - 12227-010 - São José dos Campos - SP, Brasil \\ forti@cpte.inpe.br
}

\begin{abstract}
This study compared evapotranspiration estimates (for different time scales 1,5, 10 and 30 days) from the models developed by Thornthwaith (which needs only the mean daily air temperature) and Penman-Monteith (which requires a number of climatic variables). This latter is adopted by FAO as the standard method although the former is very simple and practice to use. The both methods are computed using data from 2 years period (2007 and 2008) made at UNITAU/INMET and INMET meteorological station at Taubaté city. The Thorthwaith's method presents higher values during the hot and humid seasons (spring and summer) being the opposite for the cold and dry seasons (autumn and winter). The 10 days time scale seems to be a useful timescale for estimates of loss of water for irrigation studies.
\end{abstract}

Palavras-chave: Simulation climate, precipitation, water, simulação climática, precipitação, água.

\section{Introdução}

As reservas de água potável do mundo estão ameaçadas por fatores que incluem o crescimento demográfico, o aquecimento global, técnicas de irrigação irresponsáveis e desperdício, etc. Se nada for feito, as consequências podem ser uma crise hídrica global e instabilidades políticas. Segundo a Organização das Nações Unidas, em 20 anos faltará água para $60 \%$ do planeta. Essa previsão foi feita pela ONU com base no consumo mundial do produto. A organização alerta os consumidores para o problema, tentando levá-los a aderir à luta em favor da preservação e contra o desperdício da água (PORTAL ODM, 2009). Sendo assim, é importante se conhecer os componentes do Ciclo Hidrológico de uma região e/ou Bacia Hidrográfica. Os principais componentes deste ciclo são a entrada de água no sistema (medida pela precipitação) e a saída de água (medida ou estimada pela evapotranspiração). No caso de medidas diretas, estas são de difícil realização, uma vez que dependem de instrumentos sofisticados (por exemplo lisímetros ou sensores de resposta rápida para medidas da perda de água pela técnica da correlação de vórtices). Portanto, as estimativas da perda d'agua por métodos agrometeorológicos tentam suprir esta necessidade.

O regime pluviométrico da região já foi detalhamente analisado por Folhes e Fisch (2006). Para estimar a evapotranspiração são utilizados neste trabalho dois métodos diferentes, sendo um de natureza mais empírica e dependente somente da temperatura do ar (evapotranspiração pelo método de Thorthwaite) e outro mais realista, com a inclusão explícita dos principais elementos climáticos (radiação solar, ventos, temperatura do ar e déficit de pressão de vapor) que é estimado pelo método de Penman-Monteith, descritos anteriormente. Porém, empiricismo não é sinônimo de falta de qualidade. Muitos métodos desenvolvidos e calibrados localmente (simples) produzem melhores resultados do que aqueles mais genéricos e fisicamente mais reais (complexos). Métodos empíricos são 
resultantes entre a evapotranspiração medida em condições padronizadas e os elementos meteorológicos medidos em postos também padrões (PEREIRA, 1997). Contudo, dados de temperatura e precipitação não são suficientes para que se tenha um resultado próximo do real. Considera-se neste trabalho como o método de Penman-Monteith como o mais próximo do real devido à utilização de vários outros parâmetros, o que o torna o método mais realista e, portanto, aquele que deve ser encontrado para o balanço hídrico.

Nesse sentido, este trabalho foi realizado com o intuído de se calibrar um modelo simples de estimativa da evapotranspiração para a região de Taubaté, situado no Vale do Rio Paraíba do Sul no Estado de São Paulo. Para tanto, será utilizado o método de Thornthwaith para calcular a evapotranspiração e comparado com o método Penman-Monteith, sabendo-se que este último, por obter dados locais mais precisos e incluir explicitamente todas as variáveis meteorológicas que atuam no processo de evaporação, é o um método mais eficiente para o cálculo de evapotranspiração de uma cultura.

\section{Revisão Bibliográfica}

Neste trabalho será utilizado dois métodos de evapotranspiração: Thornthwaith, de que necessita somente do valor médio mensal da temperatura média do ar e Penman-Monteith, que necessita de uma série de variáveis que possibilitam o seu resultado ser o mais próximo do real, a saber:

\subsection{Método de Thornthwaith}

Este método foi proposto por Thornthwaith (1948) para estimativa da evapotranspiração potencial (ETP) mensal de um gramado (posto meteorológico) como um elemento climatológico, visando a classificação climática. Nesse contexto, ETP é tida como igual à "chuva ideal" para que uma região não apresente nem excesso nem deficiência hídrica durante o ano (THORNTHWAITH, 1948).

A evapotranspiração potencial média mensal padrão para um mês de 30 dias, com 12 horas de fotoperíodo, pode ser representada pelo conjunto de equações:

$$
\begin{aligned}
& \text { ETP }=16 \cdot\left(\frac{10 T i}{I}\right)^{a} \quad \text { unidades de } \mathrm{mm} / \mathrm{mês} \\
& a=6,75 \cdot 10^{-7} \cdot I^{3}-7,71 \cdot 10^{-5} \cdot I^{2}+1,7912 \cdot 10^{-2} \cdot I+0,49239 \\
& I=\sum_{i=1}^{12}(0,2 \cdot T i)^{1,514}
\end{aligned}
$$

em que $T i$ é a temperatura média mensal $\left({ }^{\circ} \mathrm{C}\right), I$ é o índice de calor da região (adimensional) e que deve ser calculado com valores normais (média climatológica) e $i$ representa o mês do ano. Porém como o número de horas por dia muda com a latitude e existem meses que variam de 28 a 31 dias, sendo então necessário adicionar um fator de correção (f):

$$
(f)=\frac{h}{12} \times \frac{n}{30}
$$

onde $h$ é o número de horas de luz solar na latitude requerida e $n$ é o número de dias do mês em estudo.

\subsection{Método de Penman-Monteith}

Para esse método a vegetação (dossel) é representada por uma enorme folha (hipótese da Big Leaf) proposta por Monteith (1965). Esta premissa baseia-se em que todas as folhas estão expostas às mesmas condições ambientais, embora essa não seja a condição real. Admite-se 
um valor médio (efetivo) que seja representativo do dossel e que, por sua vez, determina uma "transpiração média".

Em 1990, a FAO (órgão da ONU para a questão de alimentar global) reuniu um grupo de 14 especialistas em evapotranspiração para rever seu conceito de evapotranspiração de referência (DOORENBOS \& PRUITT, 1977). Dessa reunião definiu-se o método de PenmanMonteith como o mais adequado para estimar a evapotranspiração de uma cultura na escala diária, a saber:

$$
\text { ETo }=\frac{0,408 \cdot s \cdot(R n-G)+\gamma \cdot\left(\frac{900}{T+273}\right) \cdot U_{2} \cdot\left(e_{s}-e_{a}\right)}{s+\gamma \cdot\left(1+0,34 \cdot U_{2}\right)}
$$

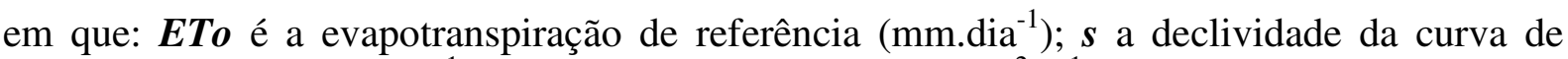
pressão de vapor $\left(\mathrm{kPa} .{ }^{\circ} \mathrm{C}^{-1}\right) ; \boldsymbol{R} \boldsymbol{n}$ o saldo da radiação $\left(\mathrm{MJ} \cdot \mathrm{m}^{-2} \cdot \mathrm{d}^{-1}\right) ; \boldsymbol{G}$ o fluxo de calor no solo $\left(\mathrm{MJ} . \mathrm{m}^{-2} \cdot \mathrm{d}^{-1}\right) ; \boldsymbol{\gamma}$ o coeficiente psicométrico $\left(\mathrm{kPa} .{ }^{\circ} \mathrm{C}^{-1}\right) ; \boldsymbol{T}$ a temperatura média diária do ar $\left({ }^{\circ} \mathrm{C}\right)$; $\boldsymbol{U}_{2}$ a velocidade do vento a $2 \mathrm{~m}$ de altura $\left(\mathrm{m} . \mathrm{s}^{-1}\right) ; \boldsymbol{e}_{\boldsymbol{s}}$ a pressão de saturação de vapor d'água à temperatura do ar (kPa) e $\boldsymbol{e}_{\boldsymbol{a}}$ a pressão atual de vapor d'água (kPa) (ANGIOLELLA, 2003).

\section{Materiais e Métodos}

O conjunto de dados existente pode ser dividido em: a) dados de temperatura do ar (escala de tempo diário) obtidos no posto meteorológico da UNITAU/INMET e b) dados horários dos principais elementos climáticos (temperatura do ar, precipitação, ventos, umidade relativa do ar, radiação solar, etc) coletados na estação automática de Taubaté (INMET - Instituto Nacional de Meteorologia, A728) no período de 2007 e 2008. Durante o ano de 2008, ocorreu falta de dados da estação automática entre 08/03 a 24/04 e de 01/08 a 24/08, representando um total de 9,85\% dos dados de calibração diários. O ano de 2007 não apresentou falta de dados.

Para calibrar os valores de Thornthwaith, partiu-se do princípio que os resultados diferenciam-se para cada estação do ano, considerando-se a primavera o período compreendido entre 23/09 a 21/12, o verão de 22/12 a 20/03, o outono de 21/03 a 20/06 e o inverno de 21/06 a 22/09.

Em cada estação do ano plotou-se os resultados Thornthwaith X Penman-Monteith com a melhor reta de ajuste para esse conjunto. A reta de regressão linear foi ajustada pela seguinte expressão:

$$
E V P_{\text {Penman-Monteith }}=a \cdot E V P_{\text {Thorthwaite }}+b
$$

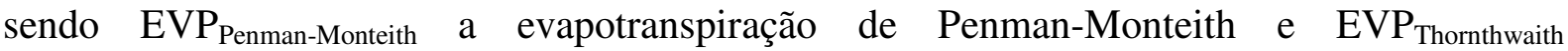
evapotranspiração de Thornthwaith. As constantes $a$ e $b$ são respectivamente, o coeficiente angular e o coeficiente linear da reta de ajuste.

\section{Resultados e Discussões}

A Figura 1 mostra as séries temporais dos resultados de evapotranspiração pelos métodos de Thorthwaite e Penman-Monteith para as escalas de tempo de 1 dia (diário), 5 dias (pêntada), 10 dias (decêndio) e 30 dias (mensal) para o período de 2007 e 2008. Observou-se que houve um acompanhamento sazonal da curva de evapotranspiração de acordo com o ciclo anual de 


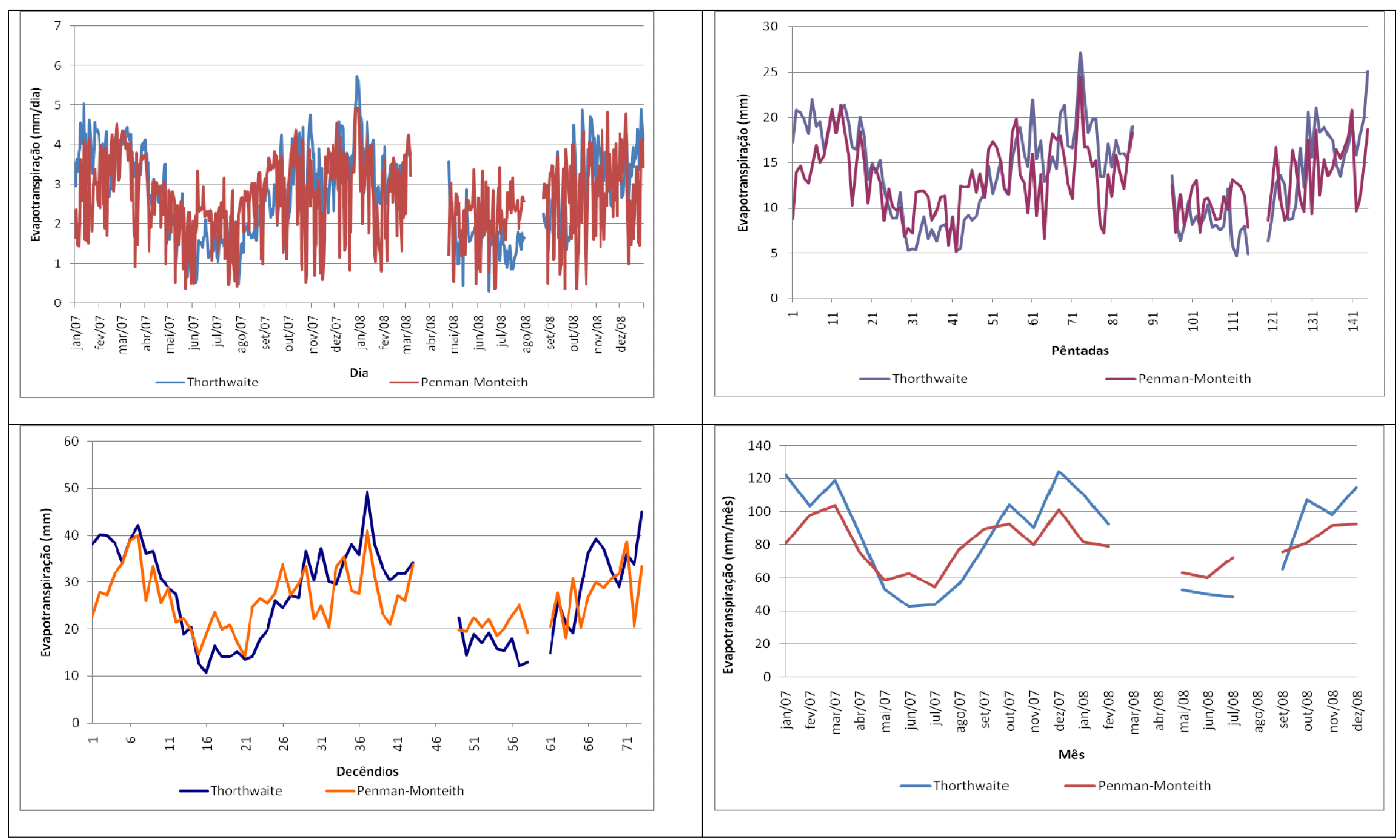

Figura 1 - Evapotranspiração pelo método de Thorthwaith em comparação com o de Penman-Monteith durante os anos de 2007 e 2008 , para as escalas de tempo diária (a), pêntada (b), decêndio (c) e mensal (d). 
radiação solar. Isto ocorreu devido a utilização, nos dois métodos, da energia proveniente do Sol: pelo método de Penman-Monteith, usou-se a radiação solar estimada a partir da medida direta da radiação solar, enquanto que por Thorthwaith os dados de temperatura do ar representaram a quantidade de energia solar incidente na superfície terrestre, transformada em calor na atmosfera.

Conforme se observa na Figura 2, os valores relativamente baixos de evapotranspiração (40 a $70 \mathrm{~mm} / \mathrm{mês}$ ), ou seja, de períodos frescos (outono e inverno), possuem uma estimativa de Thorthwaith um pouco abaixo de Penman-Monteith, diferentemente dos períodos quentes (primavera e verão), em que a evapotranspiração é maior (70 a $130 \mathrm{~mm} / \mathrm{mês}$ ), possuindo valores de Thorthwaith maior do que Penman-Monteith. Esta observação mostra que o método de Thorthwaith subestima Penman-Monteith durante o inverno e outono, e superestima durante o verão e primavera.

Na Figura 2 encontra-se os gráficos das dispersões dos dados de evapotranspiração Thorthwaite X Penman-Monteith com a sua reta de regressão linear para 2007 e 2008.

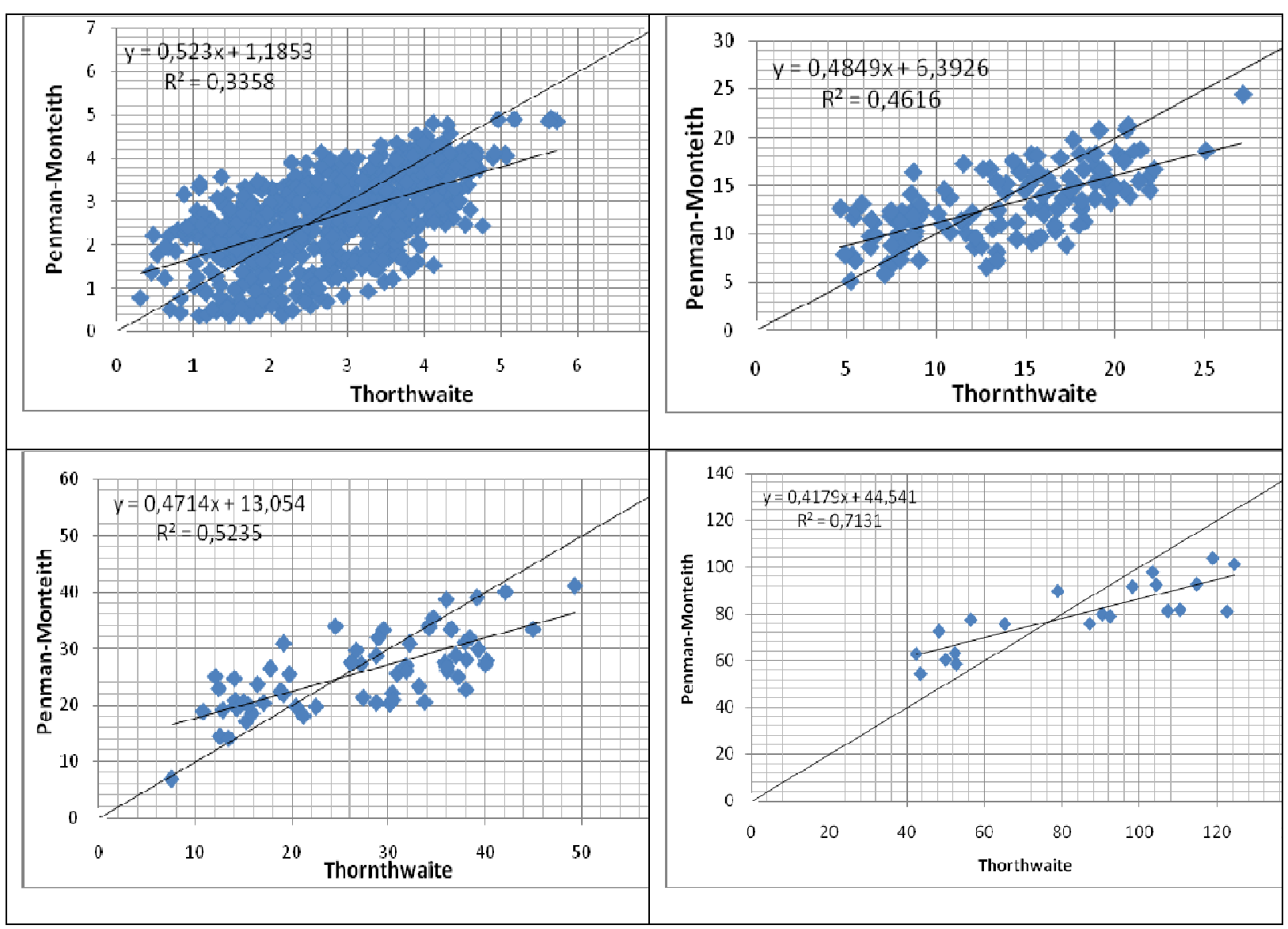

Figura 2 - Comparação das estimativas de Thorthwaite e Penman-Monteith para as escalas de tempo diária (a), pêntada (b), decêncido (c) e mensal (d). 
As Figuras 3, 4 e 5 mostram os resultados da dispersão dos dados para diferentes escalas de tempo (1, 5 e 10 dias) para as quatro estações do ano.
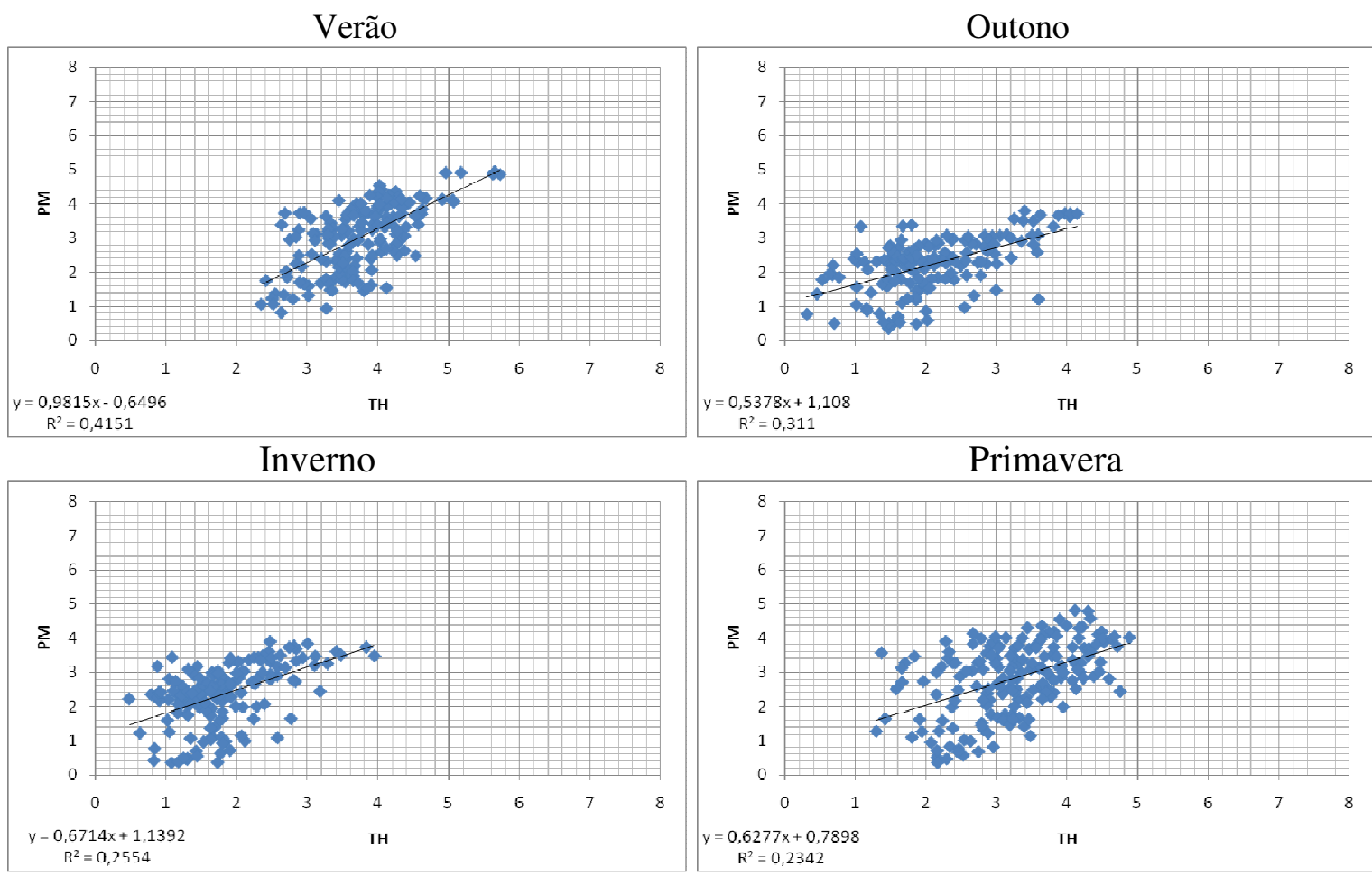

Figura 3- Dispersão das evapotranspiração determinados por Thorthwaite (TH) e PenmanMonteith (PM) para as quatro estações do ano para escala de tempo diária.

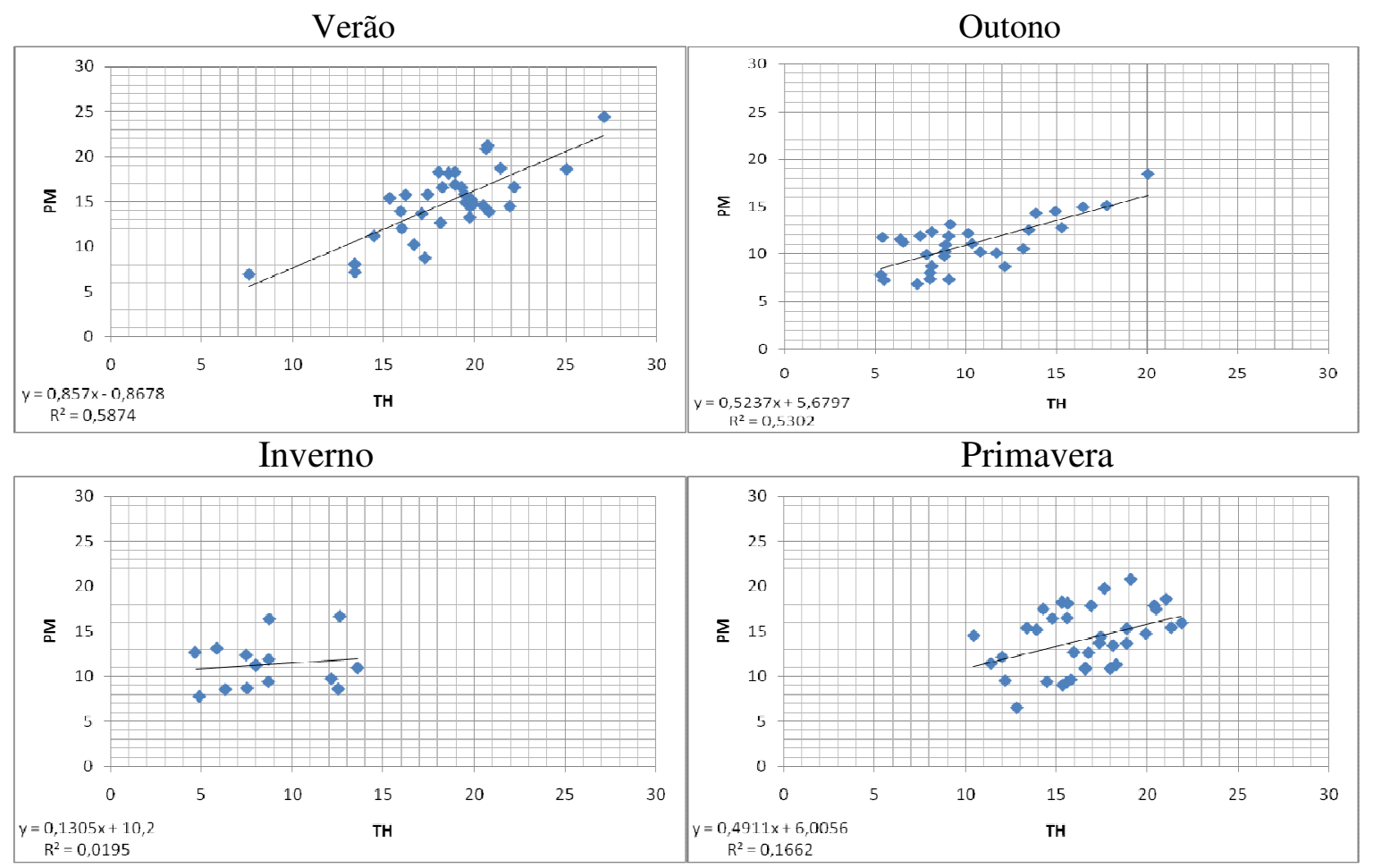

Figura 4- Dispersão das evapotranspiração determinados por Thorthwaite (TH) e PenmanMonteith (PM) para as quatro estações do ano para escala de tempo pêntada 


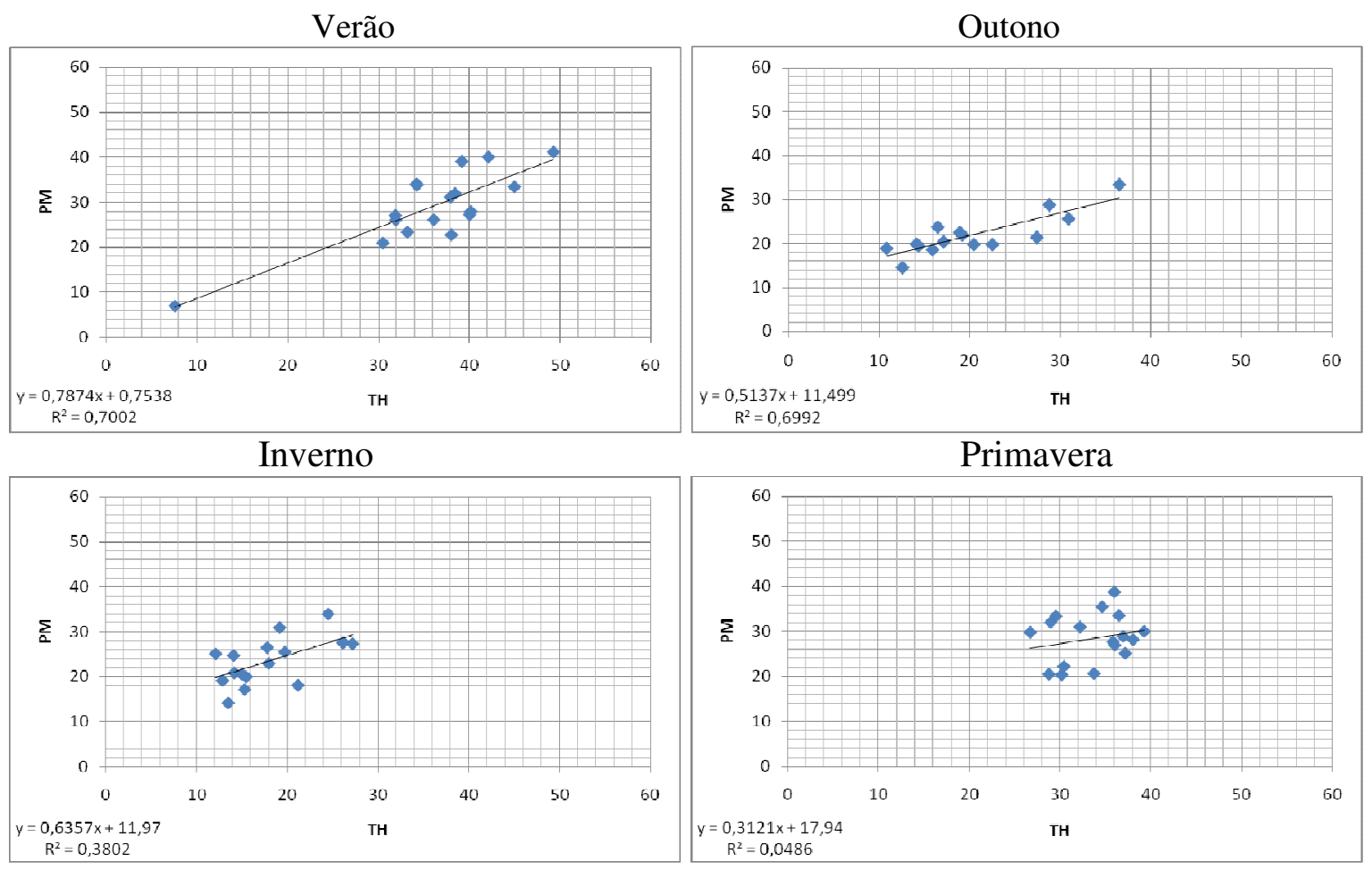

Figura 5- Dispersão das evapotranspiração determinados por Thorthwaite (TH) e PenmanMonteith (PM) para as quatro estações do ano para escala de tempo decêndio.

A tabela 1 sintetiza os resultados das comparações entre as duas estimativas através dos parâmetros das variáveis da reta de regressão linear (coeficiente de correlação, coeficientes a e b), para as diferentes escalas de tempo. A escala de tempo mensal foi realizada somente para o conjunto total de dados, uma vez que a análise por estação do ano ter-se-ia somente poucos valores.

Tabela 1 - Resultados dos parâmetros de ajuste para as diferentes escalas de tempo.

\begin{tabular}{|c|c|c|c|c|c|c|c|c|c|c|c|c|}
\hline estação & \multicolumn{3}{|c|}{ diario } & \multicolumn{3}{|c|}{ pentada } & \multicolumn{3}{|c|}{ decendio } & \multicolumn{3}{|c|}{ mensal } \\
\hline Verão & 0,98 & $-0,65$ & 41,5 & 0,86 & $-0,87$ & 58,7 & 0,79 & 0,75 & 70,0 & & & \\
\hline Outono & 0,54 & 1,11 & 31,1 & 0,52 & 5,68 & 53,0 & 0,51 & 11,50 & 69,9 & & & \\
\hline Inverno & 0,36 & 0,99 & 16,4 & 0,13 & 10,20 & 2,0 & 0,64 & 11,97 & 38,0 & & & \\
\hline Primavera & 0,37 & 2,19 & 23,4 & 0,49 & 6,01 & 16,6 & 0,31 & 17,94 & 4,9 & & & \\
\hline Todos & 0,64 & 1,09 & 32,3 & 0,49 & 6,35 & 45,8 & 0,48 & 12,83 & 52,9 & 0,42 & 44,54 & 71,3 \\
\hline
\end{tabular}




\section{Conclusão}

Observou-se, neste trabalho, que ambos os métodos estão fortemente relacionados com o ciclo sazonal da energia solar para estimar a evapotranspiração. No entanto, a estimativa pelo método de Thornthwaith superestimou a sua equivalente estimada pelo método de PenmanMonteith, nos meses quentes e chuvosos (primavera e verão) e subestimou nos meses frios e secos (outono e inverno). Este fato pode estar associado com o tipo de regime pluviométrico: nos meses de primavera e verão as chuvas são de origem convectiva ao passo que nos meses de outono e inverno as chuvas são predominantemente de origem frontal. Com relação a escala de tempo, os resultados mostraram que as correlações são melhores quanto maior o tempo. No entanto, como um compromisso entre uma boa estimativa da evapotranspiração e a prática agrícola de irrigação, sugere-se o uso da escala de tempo de 10 dias.

\section{Referências}

ANGIOLELlA, G. D.; VASCONCELlOS, V. L. D. Planilha eletrônica para cálculo do balanço hídrico climatológico normal utilizando diferentes métodos de estimativa da evapotranspiração potencial. Santa Maria: Revista Brasileira de Agrometeorologia, v.11, n.2, p. 375-378, 2003.

DOORENBOS, J.; PRUITT, W. O. Guidelines for predicting crop water requirements. Roma: FAO Irrigation and Drainage Paper 24, ed. 2, p. 179, 1977.

FOLHES, M.T.; FISCH, G. Caracterização climática e estudo de tendências nas séries temporais de temperatura do ar e precipitação em Taubaté (SP). Revista Ambi-Água, Taubaté, v. 1, n. 1, p. 61-71, 2006.

MONTEITH, J. L. Evaporation and environmental. In: SYMP. SOC. EXPL. BIOL. p. 205234, 1965.

PEREIRA, A. R., VILLA NOVA, N. A., SEDIYAMA, G. C. Evapo(transpi)ração. Piracicaba: FEALQ, p. 9-75, 1997.

PORTAL ODM. Fórum de Istambul termina sem reconhecer água como 'direito humano'. Disponível em: <http://www.portalodm.com.br/noticias.php?id_noticia=51>. Acesso em: 16 mar. 2009.

THORNTHWAITH, C. W. An approach toward a rational classification of climate. Geography Review, p. 55-94, 1948. 